\title{
Fundamental Solution of the Volkov Problem (Characteristic Representation)
}

\author{
A.A. BORGHARDT and D.Ya. KARPENKO
}

Donetsk Physical-Technical Institute, Ukrainian National Academy of Sciences, 340114 Donetsk, Ukraine

E-mail: borg@host.dipt.donetsk.ua

Received June 10, 1998; Accepted July 13, 1998

\begin{abstract}
The characteristic representation, or Goursat problem, for the Klein-Fock-Gordon equation with Volkov interaction [1] is regarded. It is shown that in this representation the explicit form of the Volkov propagator can be obtained. Using the characteristic representation technique, the Schwinger integral [2] in the Volkov problem can be calculated.
\end{abstract}

\section{Introduction}

The Klein-Fock-Gordon (KFG) equation is the basic equation of relativistic quantum mechanics (RQM). From the Cauchy problem point of view this equation has the advanced or retarded Green function as well as the causal or Feynman Green function $\Delta_{C}$. In this paper our attention is focused on the function $\Delta_{C}$, i.e.,

$$
\Delta_{C}=\frac{1}{2}\left(\Delta_{S}+i \Delta^{(1)}\right)
$$

where $\frac{1}{2} \Delta_{S}$ is the real part of the Feynman propagator $\Delta_{C}$, and is written in the form

$$
\Delta_{S}=\frac{1}{2 \pi}\left(\delta\left(\lambda^{2}\right)-\theta\left(\lambda^{2}\right) k_{0} \frac{J_{1}\left(k_{0} \lambda\right)}{\lambda}\right) .
$$

The following notation is used: $\lambda=\sqrt{c^{2} t^{2}-r^{2}}=\sqrt{x_{\mu}^{2}}$ is the 4-D interval of Minkowski space $M_{(+)}^{4}, \theta$ is the Heaviside step function, $k_{0}=\mu c / \hbar$ is the inverse Compton wave length, and $J_{1}$ is the Bessel function of the first kind with index 1 .

The function $\Delta^{(1)}$ is the imaginary part of the function $\Delta_{C}$, i.e.,

$$
\Delta^{(1)}=\frac{k_{0}}{4 \pi}\left\{\theta\left(\lambda^{2}\right) \frac{N_{1}\left(k_{0} \lambda\right)}{\lambda}+\frac{2}{\pi} \theta\left(\widetilde{\lambda}^{2}\right) \frac{K_{1}\left(k_{0} \tilde{\lambda}\right)}{\widetilde{\lambda}}\right\},
$$


$\tilde{\lambda}=\sqrt{r^{2}-c^{2} t^{2}}=\sqrt{-x_{\mu}^{2}}$ is the 4-D interval of Minkowski space $M_{(-)}^{4}(\tilde{\lambda} \geq 0), N_{1}$ is the Bessel function of the second kind, and $K_{1}$ is the MacDonald function.

In free case from, the function $\Delta_{C}$ can be obtained for all propagators RQM.

It should be noted that the explicit form of the function $\Delta_{C}$ is known for the free KFG equation only. The causal propagator $\Delta_{C}$ has not been calculated, even for the simplest interaction which is the Volkov interaction.

The purpose of this letter is to calculate the Green function for the Volkov interaction from the CR viewpoint and to seek the function $\Delta_{C}$ with the Volkov interaction from Cauchy viewpoint.

\section{Free KFG equation from CR viewpoint}

In the $\mathrm{CR}$ viewpoint we require an additional spatial coordinate. For the sake of definition, let $z$ be this coordinate. The wave equation may then be rewritten in the form

$$
\left(\frac{\partial^{2}}{\partial z^{2}}-\frac{1}{c^{2}} \frac{\partial^{2}}{\partial t^{2}}-\hat{a}^{2}\right) \Psi\left(x_{1}, x_{2}, z, t\right)=0,
$$

where the operator parameter $\hat{a}^{2}$ does not contain second derivatives with respect to $t$ and $z$.

For the KFG equation the operator parameter $\hat{a}^{2}$ is given by

$$
\hat{a}^{2}=k_{0}^{2}-\Delta_{\perp}, \quad \Delta_{\perp}=\frac{\partial^{2}}{\partial x_{1}^{2}}+\frac{\partial^{2}}{\partial x_{2}^{2}} .
$$

If $\hat{a}^{2}$ is a positive constant we obtain the 1-D telegraph equation

$$
\left(\hat{L}-a^{2}\right) \Psi(z, t)=0
$$

where $\hat{L}=\frac{\partial^{2}}{\partial z^{2}}-\frac{1}{c^{2}} \frac{\partial^{2}}{\partial t^{2}}=-4 \frac{\partial^{2}}{\partial \xi \partial \eta}$ is the 1-D wave operator and $\xi=c t-z, \eta=c t+z$ are the characteristics of a wave equation.

The fundamental solution for equation (2.3) is well known. It is called the Riemann function [4]

$$
\Psi(z, t)=J_{0}\left(\sqrt{\left(c^{2} t^{2}-z^{2}\right) a^{2}}\right)=J_{0}\left(\sqrt{\xi \eta a^{2}}\right),
$$

where $J_{0}$ is the Bessel function.

We are interested in the CR for the KFG equation (see Refs. [3, 4] and references therein). In this case equation (2.4) has the form

$$
\Psi\left(x_{1}, x_{2}, z, t\right)=J_{0}\left(\sqrt{\left(c^{2} t^{2}-z^{2}\right) \hat{a}^{2}}\right)|0\rangle,
$$

where we take the operator parameter from equation (2.2). Here $|0\rangle$ is the initial value of the wave function along the characteristics $|t|=|z| / c$ (or $c t \pm z=0$ ).

The solution (2.5) is stable, provided that the value under the square root is positive. Otherwise, if the value under the square root is negativ, the solution will be increasing. 
Equation (2.5), with the operator $\hat{a}^{2}$, should be considered in two regions of the Minkowski space $M^{2}$. The space $M^{2}$ consist of the space $M_{(+)}^{2}$, where $c^{2} t^{2}-z^{2} \geq 0$ (or $|t| \geq|z| / c)$, and the space $M_{(-)}^{2}$, where $c^{2} t^{2}-z^{2} \leq 0$ (or $\left.|t| \leq|z| / c\right)$.

We first consider the region $c^{2} t^{2}-z^{2} \geq 0$. As the initial value $|0\rangle$ we select the 2-D $\delta$-function, namely when $|t|=|z| / c$. We have the initial localized function [3]

$$
|0\rangle=\frac{1}{4 \pi^{2}} \int \exp \left(i k_{1} x_{1}+i k_{2} x_{2}\right) d k_{1} d k_{2}=\delta\left(x_{1}, x_{2}\right)
$$

Substituting equation (2.6) into equation (2.5), and integrating over the polar angle with the help of the formula

$$
\int_{0}^{2 \pi} \exp (i k x \cos (\varphi-a)) d \varphi=2 \pi J_{0}(k x)
$$

we get the fundamental solution in the $\mathrm{CR}$ in the space $M_{(+)}^{2}$. Therefore, we denote the wave function of equation $(2.5)$ as $\Psi^{(+)}$

$$
\Psi^{(+)}\left(x_{1}, x_{2}, z, t\right)=\frac{1}{2 \pi} \int_{0}^{\infty} J_{0}\left(\sqrt{\left(c^{2} t^{2}-z^{2}\right)\left(k^{2}+k_{0}^{2}\right)}\right) J_{0}\left(k x_{\perp}\right) k d k
$$

where $x_{\perp}=\sqrt{x_{1}^{2}+x_{2}^{2}}$.

It is easy to calculate the integral (2.7) if $k_{0} \rightarrow 0$. In this case we have the photon propagator [3]

$$
\left.\Psi^{(+)}\right|_{k_{0} \rightarrow 0}=\frac{\delta\left(\sqrt{c^{2} t^{2}-z^{2}}-x_{\perp}\right)}{2 \pi x_{\perp}}=\frac{\delta(|t|-r / c)}{2 \pi c r}=\frac{1}{\pi} \delta\left(\lambda^{2}\right) .
$$

For $k_{0} \neq 0$, we calculate the equation (2.7) with the help of the Sonin discontinuous integral

$$
\int_{0}^{\infty} k^{n+1} \frac{J_{m}\left(\tau \sqrt{k^{2}+k_{0}^{2}}\right)}{\left(k^{2}+k_{0}^{2}\right)^{m / 2}} J_{n}\left(k x_{\perp}\right) d k=\theta\left(\tau-x_{\perp}\right) \frac{x_{\perp}^{n}}{\tau^{m}}\left(\frac{\lambda}{x_{\perp}}\right)^{m-n-1} J_{m-n-1}\left(k_{0} \lambda\right),
$$

where $\lambda=\sqrt{\tau^{2}-x_{\perp}^{2}}$, and $m>n>0$.

Note that the integral (2.8) cannot be used directly, because in equation (2.7) numbers $m=n=0$. It is therefore necessary to increase the order of the Bessel function. In order to achieve this we use the equality from the Bessel function theory

$$
J_{0}\left(\tau \sqrt{k^{2}+k_{0}^{2}}\right)=\frac{1}{\tau} \frac{d}{d \tau}\left(\tau \frac{J_{1}\left(\tau \sqrt{k^{2}+k_{0}^{2}}\right)}{\sqrt{k^{2}+k_{0}^{2}}}\right)
$$


Substituting equation (2.9) into equation (2.7), and taking into account equation (2.8) at $m=1$ and $n=0$, we obtain the fundamental solution of the KFG equation in the space $M_{(+)}^{2}$ as

$$
\begin{aligned}
\Psi^{(+)} & =\frac{1}{2 \pi}\left(\frac{\delta\left(\tau-x_{\perp}\right)}{x_{\perp}}-k_{0} \theta\left(\tau-x_{\perp}\right) \frac{J_{1}\left(k_{0} \lambda\right)}{\lambda}\right) \\
& =\frac{1}{\pi}\left(\delta\left(\lambda^{2}\right)-\frac{k_{0}}{2} \theta\left(\lambda^{2}\right) \frac{J_{1}\left(k_{0} \lambda\right)}{\lambda}\right),
\end{aligned}
$$

where $\tau=\sqrt{c^{2} t^{2}-z^{2}}=\sqrt{\xi \eta}$ is the timelike interval of the space $M_{(+)}^{2}$.

The fundamental solution (2.10) from the CR viewpoint coincides with the function $\Delta_{S}$ of equation (1.1) from the Cauchy problem viewpoint.

We should also consider the fundamental solution in the space $M_{(-)}^{2}$, where $z^{2}-c^{2} t^{2} \geq 0$ (or $|t| \leq|z| / c$ ). For this purpose we rewrite equation (2.5) [4] as

$$
\Psi\left(x_{1}, x_{2}, z, t\right)=J_{0}\left(\sqrt{\left(z^{2}-c^{2} t^{2}\right)\left(\Delta_{\perp}-k_{0}^{2}\right)}\right)|0\rangle .
$$

We denote the wave function of equation (2.11) in the space $M_{(-)}^{2}$ as $\Psi^{(-)}$. It should be noted that the initial value $|0\rangle$, on the characteristics $|t|=|z| / c$, cannot be selected in the localized form since we obtain unstable solutions [3]. Therefore we take the superposition of the MacDonald functions $K_{0}[3]$

$$
|0\rangle=\int_{k_{0}}^{\infty} K_{0}\left(k x_{\perp}\right) k d k=k_{0} \frac{K_{1}\left(k_{0} x_{\perp}\right)}{2 \pi x_{\perp}} \underset{k_{0} \rightarrow 0}{\longrightarrow} \frac{1}{2 \pi x_{\perp}^{2}} .
$$

Taking into account the equality on eigenfunctions $\Delta_{\perp} K_{0}=k^{2} K_{0}$, and substituting equation (2.12) into equation (2.11), we obtain the fundamental solutions of the KFG equation in the space $M_{(-)}^{2}[3]$ as

$$
\begin{aligned}
& \Psi^{(-)}\left(x_{1} . x_{2}, z, t\right)=\frac{1}{2 \pi} \int_{k_{0}}^{\infty} J_{0}\left(\sqrt{\left(z^{2}-c^{2} t^{2}\right)\left(k^{2}-k_{0}^{2}\right)}\right) K_{0}\left(k x_{\perp}\right) k d k \\
& =\frac{k_{0}}{2 \pi} \frac{K_{1}\left(k_{0} \widetilde{\lambda}\right)}{\widetilde{\lambda}} \underset{k_{0} \rightarrow 0}{\longrightarrow} \frac{1}{2 \pi} \frac{1}{\widetilde{\lambda}^{2}}>0 .
\end{aligned}
$$

The function $\Psi^{(-)}$of equation (2.13), in the CR viewpoint, does not coincide with the $\Delta^{(1)}$ function (see equation (1.2)).

\section{Volkov problem from CR viewpoint}

As an example of the CR application, we regard the KFG equation with electromagnetic interaction

$$
\left(\hat{L}+\Delta_{\perp}-k_{0}^{2}+\frac{2 i e}{\hbar c} A_{\mu} \partial_{\mu}-\frac{e^{2}}{\hbar^{2} c^{2}} A_{\mu}^{2}\right) \Psi=0,
$$


where Volkov potential has the form

$$
A_{\mu}=\left(A_{1}(\xi), A_{2}(\xi), 0,0\right), \quad \xi=c t-z .
$$

The wave function of equation (3.1), with the potential (3.2), can be expanded into a Fourier integral over transverse coordinates $x_{1}, x_{2}$, i.e.,

$$
\Psi\left(x_{1}, x_{2}, z, t\right)=\frac{1}{4 \pi^{2}} \int \exp \left(i k_{1} x_{1}+i k_{2} x_{2}\right) \Phi\left(k_{1}, k_{2}, z, t\right) d k_{1} d k_{2} .
$$

Taking into account the transformation (3.3), we obtain the 1-D telegraph equation with the variable parameter $K^{2}(\xi)$

$$
\left(4 \frac{\partial^{2}}{\partial \xi \partial \eta}+K^{2}(\xi)\right) \Phi\left(k_{1}, k_{2}, \xi, \eta\right)=0
$$

where

$$
K^{2}(\xi)=k^{2}+k_{0}^{2}+\frac{2 e}{\hbar c}(\boldsymbol{A} \boldsymbol{k})+\frac{e^{2}}{\hbar^{2} c^{2}} A_{\mu}^{2}, \quad k^{2}=k_{1}^{2}+k_{2}^{2} .
$$

Since the characteristic variable (in equation (3.4) is cyclic, we can seek the solution of equation (3.4) with the initial condition on the characteristics

$$
\Phi\left(k_{1}, k_{2}, 0,0\right)=1
$$

in the form

$$
\Phi=J_{0}(\sqrt{\eta f(\xi)})
$$

where the unknown function $f(\xi)$ satisfies to the equation $f^{\prime}(\xi)=K^{2}(\xi)$. Whence it follows that

$$
f(\xi)=\int_{0}^{\xi} K^{2}(z) d z .
$$

To prove equation $(3.8)$ we need the following property $\hat{L}$-operator

$$
4 \frac{\partial^{2}}{\partial \xi \partial \eta} J_{0}(\sqrt{\eta f(\xi)})=-f^{\prime}(\xi) J_{0}(\sqrt{\eta f(\xi)})
$$

We still need to calculate the integral (3.3) with the function $\Phi$, which is determined by equations (3.7) and (3.8), i.e.,

$$
\begin{aligned}
& \Psi=\frac{1}{4 \pi^{2}} \int d k_{1} d k_{2} \exp i\left(k_{1} x_{1}+k_{2} x_{2}\right) J_{0}\left(\sqrt{\xi \eta\left\langle K^{2}(\xi)\right\rangle}\right), \quad \text { and } \\
& \left\langle K^{2}(\xi)\right\rangle=\frac{1}{\xi} \int_{0}^{\xi} K^{2}(z) d z .
\end{aligned}
$$

The integral (3.10) reduces to the free case if we make the shift of the momentum

$$
\boldsymbol{q}=\boldsymbol{k}+\frac{e}{\hbar c}\langle\boldsymbol{A}\rangle \text {. }
$$


In fact, the 2-D vector $\boldsymbol{q}$ is the general momentum. After this we get the same integral as in the free case but the value $k_{0}$ is replaced by $k_{0}(\xi)$

$$
\Psi=\frac{1}{4 \pi^{2}} \exp \left(-\frac{i e}{\hbar c} x_{\mu}\left\langle A_{\mu}\right\rangle\right) \int \exp i\left(q_{1} x_{1}+q_{2} x_{2}\right) J_{0}\left(\sqrt{\xi \eta\left(q^{2}+k_{0}^{2}(\xi)\right)}\right) d q_{1} d q_{2},
$$

where

$$
k_{0}(\xi)=k_{0} \sqrt{1+\left(\frac{e}{\hbar c k_{0}}\right)^{2}\left(\left\langle A_{\mu}^{2}\right\rangle-\left\langle A_{\mu}\right\rangle^{2}\right)} .
$$

Reasoning as in the calculation of the integral (2.7), we obtain the fundamental solution of the KFG equation with the Volkov interaction from the CR viewpoint

$$
\Psi=\frac{1}{\pi} \exp \left(-\frac{i e}{\hbar c} x_{\mu}\left\langle A_{\mu}\right\rangle\right)\left\{\delta\left(\lambda^{2}\right)-\frac{k_{0}(\xi)}{2} \theta\left(\lambda^{2}\right) \frac{J_{1}\left(k_{0}(\xi) \lambda\right)}{\lambda}\right\} .
$$

The solution (3.13) allows us to conclude that the particle in the electromagnetic wave becomes more heavy. Its mass is increased by $\sqrt{1+\left(\frac{e}{\hbar c k_{0}}\right)^{2}\left(\left\langle A_{\mu}^{2}\right\rangle-\left\langle A_{\mu}\right\rangle^{2}\right)^{2}}$ times. The same conclusion follows from [5], where the Volkov problem is considered from the classical Lorentz equation, and also from [6] (page 188; The nonlinear interaction of massless spinor particle with electromagnetic field generates the mass of a particle).

The propagator of the Dirac equation with the Volkov interaction was considered in papers $[2,7,8]$ as well as in many other papers. In particular in [2] the propagator was found in the form of the proper time integral. If we neglect the spin dependence and perform the change of variable in integration $\alpha=1 / 4 s$, then the propagator determined by equation (4.26) in [2] can be represented in the form

$$
G_{s}\left(x^{\prime}, x^{\prime \prime}\right)=-\frac{1}{4 \pi^{2}} C\left(x^{\prime}, x^{\prime \prime}\right) \int_{0}^{\infty} \exp \left(\frac{-i k_{0}^{2}\left(\xi^{\prime}, \xi^{\prime \prime}\right)}{4 \alpha}-i \lambda \alpha\right) d \alpha, \quad \lambda^{2}=-x_{\mu}^{2}
$$

where

$$
k_{0}^{2}\left(\xi^{\prime}, \xi^{\prime \prime}\right)=k_{0}^{2}+\frac{1}{\xi^{\prime}-\xi^{\prime \prime}} \int_{\xi^{\prime \prime}}^{\xi^{\prime}} A_{\mu}^{2}(\xi) d \xi-\left(\frac{1}{\xi^{\prime}-\xi^{\prime \prime}} \int_{\xi^{\prime \prime}}^{\xi^{\prime}} A_{\mu}(\xi) d \xi\right)^{2} .
$$

The integral (3.14) was calculated in the appendix of Schwingers paper [9]. The real part of the integral (3.14) is equal to the expression in brackets \{\} of equation (3.13). The imaginary part of the integral (3.14) is equal to the function $\Delta^{(1)}$ of equation $(1.2)$, in which $k_{0} \rightarrow k_{0}(\xi)$.

It is important to note that the factor $C\left(x^{\prime}, x^{\prime \prime}\right)$ coincides with the factor before the integral (3.11). The latter was found by us while considering the Volkov problem from the CR viewpoint.

Thus, up to the phase factor, the Schwinger propagator (3.14) for the Volkov interaction (3.2), is the free function $\Delta_{C}$ with $k_{0}(\xi)$

$$
G_{S}\left(x^{\prime}, x^{\prime \prime}\right)=-\frac{1}{4 \pi^{2}} \exp \left(-i\left(x^{\prime}-x^{\prime \prime}\right)_{\mu} \frac{1}{\xi^{\prime}-\xi^{\prime \prime}} \int_{\xi^{\prime \prime}}^{\xi^{\prime}} A_{\mu}(\xi) d \xi\right) \Delta_{C}\left(x^{\prime}, x^{\prime \prime}, k_{0}\left(\xi^{\prime}, \xi^{\prime \prime}\right)\right)
$$




\section{References}

[1] Volkov D.M.., Zs. f. Phys., 1935, V.94, 25.

[2] Schwinger J., Phys. Rev., 1951, V.82, 664.

[3] Borghardt A.A. and Karpenko D.Ya., J. Math. Phys., 1996, V.37, 233.

[4] Borghardt A.A. and Karpenko D.Ya., JETP, 1997, V. 85(4), 635-639.

[5] Bagrov V.G. and Gitman D.M., Exact Solutions of Relativistic Wave Equations, Kluwer, Dordrecht, 1990.

[6] Fushchych W. and Zhdanov R., Symmetries and Exact Solutions of Nonlinear Dirac Equations, Mathematical Ukraina Publisher, Kyiv, 1997.

[7] Nambu Y., Progr. Theor. Phys., 1950, V.5, 82.

[8] Fock V.A., Sov. Phys., 1937, V.12, 404.

[9] Schwinger J., Phys. Rev., 1949, V.76, 790. 